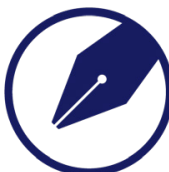

\title{
Ranking the Success Factors to Improve Safety and Growth of Artichoke Medicinal Plant Using Fuzzy Analytic Hierarchy Process
}

\author{
J. Rezaeian ${ }^{1^{*}}$, F. Talebi ${ }^{2}$, R. Alizadeh Foroutan ${ }^{1}$ \\ ${ }^{1}$ Mazandaran University of Science and Technology, Babol, Iran \\ ${ }^{2}$ Agricultural Sciences and Natural Resources University, Sari, Iran \\ E-mail: j.rezaeian@ustmb.ac.ir
}

\begin{abstract}
Today, the use of herbal medicine like artichoke is rapidly increasing worldwide. The consumption of artichoke as a food source is raising too. Therefore, it is necessary to determine the main factors affecting the growth of this plant which is investigated in this study. A comprehensive analysis of the literature is performed to detect these factors. A fuzzy Analytic Hierarchy Process (AHP) is employed to prioritize the factors. Results indicate that the conditions of irrigation and soil are the most important ones. In addition, the best conditions for artichoke growth are the medium level of irrigation, soil with suitable drainage, low temperature and sufficient sunlight.
\end{abstract}

Keywords: fuzzy analytic hierarchy process, artichoke medicinal plant, success factors

\section{Introduction and literature review}

Nowadays, the growing trend of medicinal herbs usage and the special attention of the human society to these herbs have led researchers to study and test different aspects of herbs and to improve the qualitative properties of medicinal herbs by studying the effects of various factors affecting the growth of artichoke including light, irrigation, temperature, soil, etc. These factors play a significant role in the growth and health of plants, including medicinal herbs (Figure 1).

The effects of artichoke as a medicinal plant on the human life have been studied by many researchers. Petropoulos et al. ${ }^{[1]}$ evaluated the nutritional value and chemical composition of Greek artichoke genotypes and found that the palmitic and linoleic acids are the most abundant fatty acids in artichoke heads. The vitamin $\mathrm{C}$ and fatty acids contents, chemical conversions and overall chemical composition of Jerusalem artichoke under fermentation followed by freeze-drying was studied by Koczoń et al. ${ }^{[2]}$. Diaz et al. ${ }^{[3]}$ investigated the function of underground tuber of Jerusalem artichoke in dietary fiber.

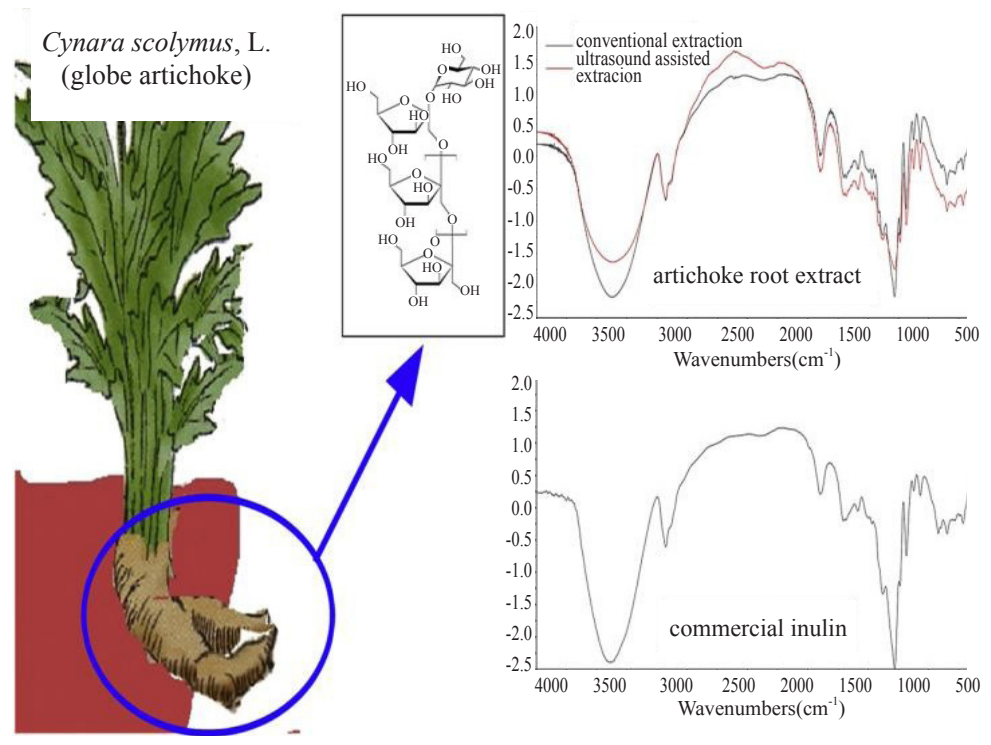

Figure 1. The suitable inulin of artichoke roots ${ }^{[13]}$

Copyright (C2020 J. Rezaeian, et al

DOI: https://doi.org/10.37256/fse.122020113

This is an open-access article distributed under a CC BY license

(Creative Commons Attribution 4.0 International License)

https://creativecommons.org/licenses/by/4.0/ 
These studies indicate the significant role of artichoke in human health and safety. Hence, identifying the factors which affect the growth and chemical composition of this plant is essential and vital. In this field, Rabara et al. ${ }^{[4]}$ studied the implementation of artificial red, blue and white LED lights on the growth of artichoke. They showed that the photosynthetic photon flux density (PPFD) has the highest effect under the red LED light which is only a third of the total PPFD compared to natural light. In addition, the red light significantly influences the height, root length, biomass and chlorophyll contents (Figure 2).

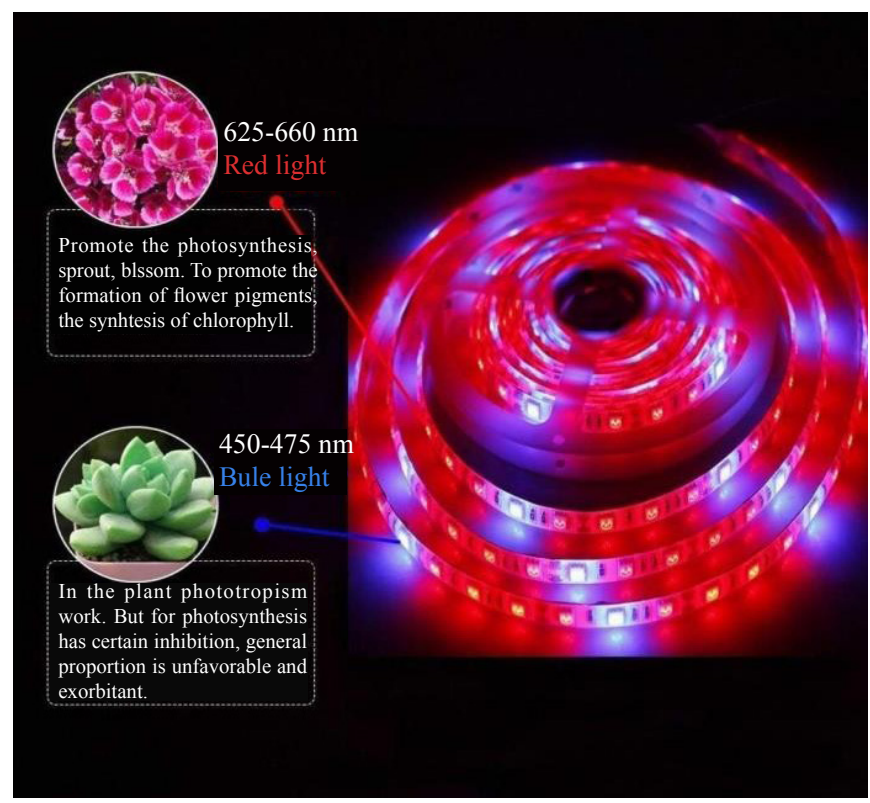

Figure 2. The effect of red light on growth of artichoke

Long et al. ${ }^{[5]}$ studied two genotypes of Jerusalem artichoke by different seawater concentrations and various periods of time. They evaluated the seedling growth, ion content, and photosynthetic productivity in a greenhouse. The results showed the reduction of almost all the growth parameters. Rouphael et al. ${ }^{[6]}$ experimented the effect of nutrient solution concentration on leaf dry biomass, leaf number, and macro element concentrations of artichoke and found a direct relationship between them. Leskovar and Othman ${ }^{[7]}$ mentioned the influence of nitrogen and fustigation methods on growth of artichoke.

Up to now, many researchers have tried to detect the effective factors on growth of artichoke under various laboratory conditions where three majority concerns are considerable. The first concern is that the experiment is time consuming and costly. The second one is that there are too many factors such that it is impossible to control all of them. And, the last one is that the features of artichoke under different conditions of breeding will be varied. Because of these concerns, use of expert systems such as knowledge-based systems, artificial intelligence, etc. can be profitable for industries. Piri et al ${ }^{[8]}$. proposed an Analytic Hierarchy Process (AHP) and Geographic Information System (GIS) for selecting the suitable areas for medicinal species. Paungbut et al. ${ }^{[1]}$ investigated growth and phenology of Jerusalem artichoke. They found that seasonal variation is influenced on phenological development and growth stages of the plant.

Due to the increasing population of the world and the importance of the increasing growth of plants, this study designs an expert-based model for identification and prioritizing the effective factors of growth of artichoke medicinal plant under the climatic and geographical conditions of cultivation in farms of the city of Sari in northern Iran. Many studies tries to detect and measure the impact of important factors on the growth of plants. Most of these studies try to do it in laboratory conditions ${ }^{[16]}$. Some of them also do it in natural situations. In this case the environmental factors such as temperature influence the growth of plant in addition of bio and chemical fertilizer ${ }^{[17]}$. Hence, the optimal set of this factors in different situations are important for researchers. In this study, the setting of the environmental conditions is done by experts which are aware of the environment as prestudy.

A fuzzy Analytic Hierarchy Process (AHP) is employed to prioritize the factors affecting the growth of artichoke, since it is a structured technique for organizing and analyzing complex decisions ${ }^{[14,15]}$. The focus of the presented study is on the therapeutic features of the plant in the treatment of liver diseases. It can be a preliminary study for researchers to strategically control the factors which are more effective and save time and cost. 


\section{Methodology}

The first and main step of this study is based on the presented literature review for detecting the effective factors in growth of artichoke. A careful study of researches is shown that some factors influence the growth of plants such as artichoke generally. For this reason, in this study these factors are identified by experts via DELPHI techniques and summarized in Table 1. Based on this table, the factors affecting the growth of artichokes are grouped in four types and sub-factors are determined for each group.

Table 1. The effective factors and sub-factors on the growth of artichokes

\begin{tabular}{cc}
\hline Factors & Sub-factors \\
\hline Light & Light intensity, sunlight \\
Irrigation quantity & Low, medium, much \\
Temperature & Low, medium, high \\
Soil & Soil depth, PH of soil, soil drainage \\
\hline
\end{tabular}

\subsection{Analytic Hierarchy Process}

Analytic Hierarchy Process (AHP), as one of the most applicable techniques for multiple-criteria decision-making (MCDM) was proposed by Saaty ${ }^{[9]}$. The main feature of this technique is pairwise comparisons between criteria and alternatives based on each criterion. To do so, a hierarchy structure of problem is constructed. The hierarchy structure of the proposed problem is shown in Figure 3.

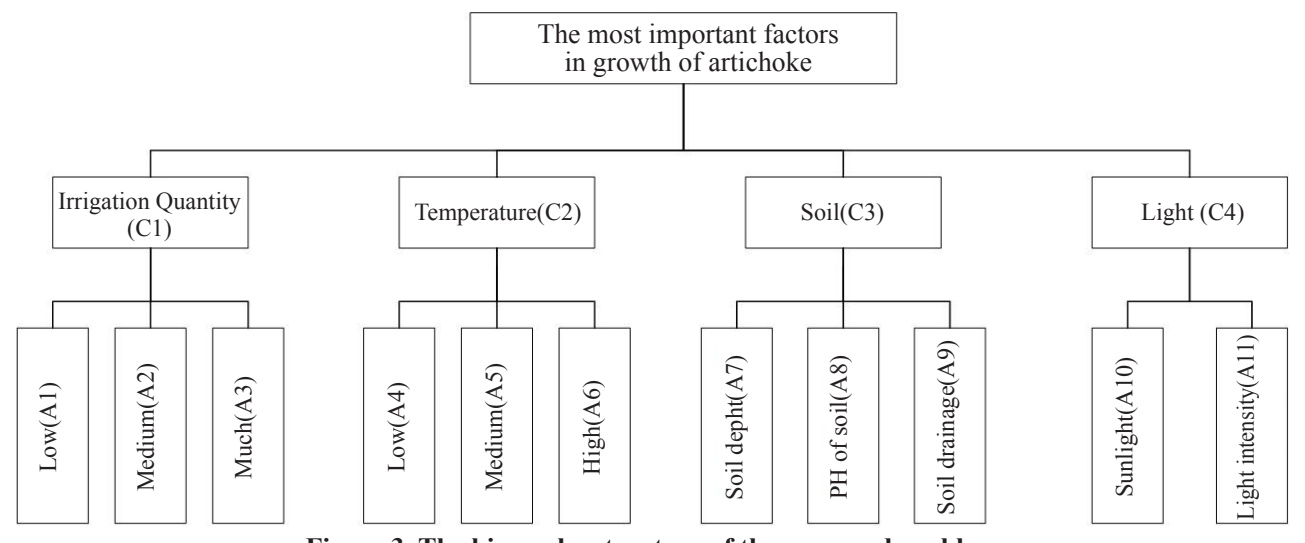

Figure 3. The hierarchy structure of the proposed problem

\subsection{Fuzzy AHP}

Fuzzy set theory was first introduced by Zadeh ${ }^{[12]}$ as a mathematical approach to represent and handle vagueness in decision-making. In this study, the fuzzy numbers equivalent to linguistic variables are set according to Table 2 .

Table 2. Linguistic variables of the proposed problem

\begin{tabular}{ccc}
\hline Fuzzy nubers & Crisp values & Linguistic variable \\
\hline$(0,0.1,0.2)$ & 1 & Equal importance (E) \\
$(0.1,0.2,0.3)$ & 2 & Weak (W) \\
$(0.2,0.3,0.4)$ & 3 & Moderate importance (MI) \\
$(0.3,0.4,0.5)$ & 4 & Moderate plus (MP) \\
$(0.4,0.5,0.6)$ & 5 & Strong importance (SI) \\
$(0.5,0.6,0.7)$ & 6 & Strong plus (SP) \\
$(0.6,0.7,0.8)$ & 7 & Very strong importance (VS) \\
$(0.7,0.8,0.9)$ & 8 & Very very strong (VV) \\
$(0.8,0.9,1)$ & 9 & Extreme importance (EI) \\
\hline
\end{tabular}




\subsection{De-fuzzification method}

In this study, the fuzzy numbers are converted into crisp values based on the method introduced by Opricovic and Tzeng ${ }^{[10]}$. In this method, $A_{i j}^{k}=\left(l_{i j}^{k}, m_{i j}^{k}, r_{i j}^{k}\right)$ indicates the fuzzy assessment between the criterion $i$ and the criterion $j$ of the $k$ th evaluator. The steps of the method are described as follow:

Step 1: Normalization:

$$
\begin{aligned}
& \Delta_{\text {min }}^{\max }=\operatorname{maxr}_{i j}^{k}-m i n l_{i j}^{k}, \\
& x l_{i j}^{k}=\frac{l_{i j}^{k}-m_{i n l_{i j}^{k}}}{\Delta_{\text {min }}^{\max }}, \\
& x m_{i j}^{k}=\frac{m_{i j}^{k}-m_{i n} l_{i j}^{k}}{\Delta_{\text {min }}^{\max }}, \\
& x u_{i j}^{k}=\frac{r_{i j}^{k}-\min _{i j}^{k}}{\Delta_{\text {min }}^{\max }} .
\end{aligned}
$$

Step 2: Computing lower (ls) and upper (us) normalized values:

$$
\begin{aligned}
& x l s_{i j}^{k}=\frac{x m_{i j}^{k}}{1+x m_{i j}^{k}-x l_{i j}^{k}}, \\
& x u s_{i j}^{k}=\frac{x r_{i j}^{k}}{1+x u_{i j}^{k}-x m_{i j}^{k}} .
\end{aligned}
$$

Step 3: Computing total normalized crisp value:

$$
x_{i j}^{k}=\frac{\left[x l s_{i j}^{k}\left(1-x l_{i j}^{k}\right)+x u s_{i j}^{k}\right]}{\left[1-x l s_{i j}^{k}+x u s_{i j}^{k}\right]} .
$$

Step 4: Computing crisp value:

$$
z_{i j}^{k}=\operatorname{minl}_{i j}^{k}+x_{i j}^{k} \Delta_{\min }^{\max } .
$$

$Z_{i j}^{k}$ is a crisp value of comparison between the criteria or alternatives $i$ and $j$ for the $k t h$ evaluator.

$$
z_{i j}=\sqrt[k]{z_{i j}^{1} \times z_{i j}^{2} \times \ldots \times z_{i j}^{k}}
$$

The final weight of each criterion or alternative can be calculated by equation (10) introduced by Saaty ${ }^{[9]}$.

$$
W_{i}=\frac{\left(\prod_{j=1}^{n} Z_{i j}\right)^{\frac{1}{n}}}{\sum_{i=1}^{n}\left(\prod_{j=1}^{n} Z_{i j}\right)^{\frac{1}{n}}}, i, j=1,2, \ldots, n .
$$


where " $n$ " is the number of criteria or alternatives in pairwise comparisons matrix.

\section{Discussion}

In this section, the steps of the proposed method are applied. The linguistic pairwise comparison of criteria and their fuzzy equivalence are done using the opinion of many experts. The results are summarized in Tables 3 and 4.

Table 3. The linguistic pairwise comparisons matrix with respect to the goal

\begin{tabular}{ccccc}
\hline Criteria & $\mathrm{C}_{1}$ & $\mathrm{C}_{2}$ & $\mathrm{C}_{3}$ & $\mathrm{C}_{4}$ \\
\hline $\mathrm{C}_{1}$ & $\mathrm{I}$ & $\mathrm{SP}$ & $\mathrm{E}$ & $\mathrm{SP}$ \\
$\mathrm{C}_{2}$ & - & $\mathrm{I}$ & $1 / \mathrm{SI}$ & $\mathrm{E}$ \\
$\mathrm{C}_{3}$ & - & - & $\mathrm{I}$ & $\mathrm{SI}$ \\
$\mathrm{C}_{4}$ & - & - & - & $\mathrm{I}$ \\
\hline
\end{tabular}

Table 4. The fuzzy pairwise comparisons matrix with respect to the goal

\begin{tabular}{ccccc}
\hline Criteria & $\mathrm{C}_{1}$ & $\mathrm{C}_{2}$ & $\mathrm{C}_{3}$ & $\mathrm{C}_{4}$ \\
\hline $\mathrm{C}_{1}$ & 1 & $(0.5,0.6,0.7)$ & $(0,0.1,0.2)$ & $(0.5,0.6,0.7)$ \\
$\mathrm{C}_{2}$ & - & 1 & $1 /(0.4,0.5,0.6)$ & $(0,0.1,0.2)$ \\
$\mathrm{C}_{3}$ & - & - & 1 & $(0.4,0.5,0.6)$ \\
$\mathrm{C}_{4}$ & - & - & - & 1 \\
\hline
\end{tabular}

From Table 4, the weights of criteria are computed as follows:

$$
\mathrm{w}_{1}=0.9855, \mathrm{w}_{2}=0.0041, \mathrm{w}_{3}=0.0101, \mathrm{w}_{4}=0.0002
$$

Then, the alternatives are compared together based on each criterion and the results are shown in Tables 5, 6, 7 and 8 .

Table 5. The fuzzy pairwise comparisons matrix with respect to the irrigation quantity (C1)

\begin{tabular}{cccc}
\hline Alternatives & $\mathrm{A}_{1}$ & $\mathrm{~A}_{2}$ & $\mathrm{~A}_{3}$ \\
\hline $\mathrm{A}_{1}$ & 1 & $1 /(0.6,0.7,0.8)$ & $1 /(0.2,0.3,0.4)$ \\
$\mathrm{A}_{2}$ & - & 1 & $1 /(0.5,0.6,0.7)$ \\
$\mathrm{A}_{3}$ & - & - & 1 \\
\hline
\end{tabular}

Based on Table 5, the value of each alternative is computed according to irrigation quantity as follows:

$$
\mathrm{w}_{11}=0.0474, \mathrm{w}_{21}=0.7625, \mathrm{w}_{31}=0.2053,
$$

Table 6. The fuzzy pairwise comparisons matrix with respect to the temperature (C2)

\begin{tabular}{cccc}
\hline Alternatives & $\mathrm{A}_{4}$ & $\mathrm{~A}_{5}$ & $\mathrm{~A}_{6}$ \\
\hline $\mathrm{A}_{4}$ & 1 & $(0.3,0.4,0.5)$ & $(0.7,0.8,0.9)$ \\
$\mathrm{A}_{5}$ & - & 1 & $(0.5,0.6,0.7)$ \\
$\mathrm{A}_{6}$ & - & - & 1 \\
\hline
\end{tabular}

Based on Table 6, the value of each alternative is computed according to temperature as follows:

$$
\mathrm{w}_{42}=0.9473, \mathrm{w}_{52}=0.0466, \mathrm{w}_{62}=0.0061 \text {, }
$$

Table 7. The fuzzy pairwise comparisons matrix with respect to the soil (C3)

\begin{tabular}{cccc}
\hline Alternatives & $\mathrm{A}_{7}$ & $\mathrm{~A}_{8}$ & $\mathrm{~A}_{9}$ \\
\hline $\mathrm{A}_{7}$ & 1 & $(0.4,0.5,0.6)$ & $(0,0.1,0.2)$ \\
$\mathrm{A}_{8}$ & - & 1 & $1 /(0.4,0.5,0.6)$ \\
$\mathrm{A}_{9}$ & - & - & 1 \\
\hline
\end{tabular}


Based on Table 7, the value of each alternative is computed according to soil as follows:

$$
\mathrm{w}_{73}=0.0042, \mathrm{w}_{83}=0.1375, \mathrm{w}_{93}=0.8583,
$$

Table 8. The fuzzy pairwise comparisons matrix with respect to the light $(\mathrm{C} 4)$

\begin{tabular}{ccc}
\hline Alternatives & $\mathrm{A}_{10}$ & $\mathrm{~A}_{11}$ \\
\hline $\mathrm{A}_{10}$ & 1 & $(0.4,0.5,0.6)$ \\
$\mathrm{A}_{11}$ & - & 1 \\
\hline
\end{tabular}

Based on Table 8, the value of each alternative is computed according to light as follows:

$$
\mathrm{w}_{104}=0.1785, \mathrm{w}_{114}=0.8215,
$$

Finally, the weight of each alternative can be computed according the values reported in Table 9:

Table 9. The final weights of alternatives

\begin{tabular}{ccccccc}
\hline Alternatives & $\mathrm{C}_{1}$ & $\mathrm{C}_{2}$ & $\mathrm{C}_{3}$ & $\mathrm{C}_{4}$ & Weight & Rank \\
\hline Criteria & 0.9855 & 0.0041 & 0.0101 & 0.0002 & - & - \\
$\mathrm{A}_{1}$ & 0.0474 & - & - & - & $0 / 046713$ & 3 \\
$\mathrm{~A}_{2}$ & 0.7625 & - & - & - & $0 / 751444$ & 1 \\
$\mathrm{~A}_{3}$ & 0.2053 & - & - & - & $0 / 202323$ & 2 \\
$\mathrm{~A}_{4}$ & - & 0.9473 & - & - & $0 / 003884$ & 5 \\
$\mathrm{~A}_{5}$ & - & 0.0466 & - & - & $0 / 000191$ & 7 \\
$\mathrm{~A}_{6}$ & - & 0.0061 & - & - & $0 / 000025$ & 11 \\
$\mathrm{~A}_{7}$ & - & - & 0.0042 & - & $0 / 000042$ & 9 \\
$\mathrm{~A}_{8}$ & - & - & 0.1375 & - & $0 / 001389$ & 6 \\
$\mathrm{~A}_{9}$ & - & - & 0.8583 & - & $0 / 008669$ & 4 \\
$\mathrm{~A}_{10}$ & - & - & - & 0.1785 & $0 / 000036$ & 10 \\
$\mathrm{~A}_{11}$ & - & - & - & 0.8215 & $0 / 000164$ & 8 \\
\hline
\end{tabular}

Table 9 shows that the weight of irrigation and the weights of its alternatives are the largest values and the soil condition and the drainage of it are the next important factors. Furthermore, the temperature and light criteria are less important.

\section{Conclusions and future work}

According to the results, it can be summarized that the medium level of irrigation, soil with suitable drainage, low temperature and sufficient sunlight are the best conditions of artichoke growth. Regarding the importance of irrigation, the identification factors, their levels of control that influence irrigation, and the interrelation between them, techniques such as Analytic Network Process (ANP) can be employed in the future. Also, determining another factors which influenced the growth of artichoke such as.... can be employed in the future.

\section{References}

[1] A. Petropoulos, C. Pereira, G. Ntatsi, N. Danalatos. L. Barros, I, Ferreirab. Nutritional value and chemical composition of Greek artichoke genotypes. Food Chemistry. 2018; 267: 296-302.

[2] P. Koczoń, T. Niemiec, B.J., Bartyzel, E. Gruczyńsk, A. Bzducha-Wróbel, P. Koczoń. Chemical changes that occur in Jerusalem artichoke silage. Food Chemistry. 2019; 295: 172-179.

[3] A. Díaz, R. Bomben, C. Dini, S.Z. Viña, M.A. García, M. Ponzi, N. Comelli. Jerusalem artichoke tuber flour as a wheat flour substitute for biscuit elaboration. LWT-Food Science and Technology. 2019; 3: 82. Available from: doi: https:// doi.org/10.1016/j.lwt.2019.03.082.

[4] R.C. Rabara, G. Behrman, T. Timbol, P.J Rushton. Effect of spectral quality of monochromatic LED lights on the growth of artichoke seedlings. Frontiers in plant science. 2017; 8: 190.

[5] X. Long, Z. Huang, Z. Zhang, Q. Li, R. Zed, Z. Liu. Seawater stress differentially affects germination, growth, hotosynthesis, and Ion concentration in genotypes of Jerusalem artichoke (Helianthus tuberosus L.). Journal of plant growth 
regulation. 2010; 29: 223-231.

[6] Y. Rouphael, M. Cardarelli, L. Lucini, E. Rea, G. Colla. Nutrient solution concentration affects growth, mineral composition, phenolic acids, and flavonoids in leaves of artichoke and cardoon. Hortscience. 2012; 47: 1424-1429.

[7] D. Leskovar, Y. Othman. Low nitrogen fertigation promotes root development and transplant quality in globe artichoke. Hortscience. 2016; 51: 567-572.

[8] I. Piri, M. Moosavi, A.Z. Taheri, H. Alipur, S. Shojaei, S.A Mousavi. The spatial assessment of suitable areas for medicinal species of astragalus (Astragalus hypsogeton Bunge) using the Analytic Hierarchy Process (AHP) and Geographic Information System (GIS). The Egyptian Journal of Remote Sensing and Space Sciences. 2019; 22: $193-201$.

[9] Saaty, L.T. The Analytic Hierarchy Process. New York: McGraw Hill Company; 1980.

[10] S. Opricovic, G.H. Tzeng. Defuzzification for a fuzzy multicriteria decision model. International Journal of Uncertainty, Fuzziness and Knowledge-based Systems. 2003; 11: 635-52.

[11] D. Paungbut, S. Jogloy, N. Vorasoot, A. Patanothai. Growth and phenology of jerusalem artichoke (Helianthus tuberosus L.). Pak. J. Bot. 2015; 47: 2207-2214.

[12] L.A. Zadeh. Fuzzy sets. Information and control. 1965; 8(3): 338-353.

[13] M. Castellino, M. Renna, B. Leoni, M. Calasso, G. Difonzo, P. Santamaria, G. Gambacorta, F. Caponio, M.D. Angelis, V.M. Paradisoa. Conventional and unconventional recovery of inulin rich extracts for food use from the roots of globe artichoke. Food Hydrocolloids. 2020; 107: 1-11.

[14] Ho, M.C., Ong, V.Z., Wu, T.Y. Potential use of alkaline hydrogen peroxide in lignocellulosic biomass pretreatment and valorization-A review. Renewable and Sustainable Energy Reviews. 2019; 112: 75-86.

[15] Li, K., Qin, J.C., Liu, C.G., Bai, F.W. Optimization of pretreatment, enzymatic hydrolysis and fermentation for more efficient ethanol production by jerusalem artichoke stalk. Bioresource technology. 2016; 221: 188-194.

[16] Allahdadi, M., Y, Raei. Growth and chlorogenic acid content of artichoke (Cynara scolymus L.) affected by bio and chemical fertilizer. Journal of Biodiversity and Environmental Sciences. 2017; 11: 63-73.

[17] Allahdadi, M. Different aspects of artichoke (Cynara scolymus L.) medicinal plant: A review. Journal of Herbal Drugs. 2019; 9: 63-71. 